\title{
A SELECTION THEOREM FOR MULTIFUNCTIONS
}

\author{
H. SARBADHIKARI
}

\begin{abstract}
In this paper the following theorem is proved. $X$ is any set, $\mathbf{H}$ is a family of subsets of $X$ which is $\lambda$-additive, $\lambda$-multiplicative and satisfies the $\lambda$-WRP for some cardinal $\lambda>\boldsymbol{\aleph}_{0}$. Suppose $Y$ is a regular Hausdorff space of topological weight $\leqslant \lambda$ such that given any family of open sets, there is a subfamily of cardinality $<\lambda$ with the same union. Let $F: X \rightarrow \mathbf{C}(Y)$, where $\mathrm{C}(Y)$ is the family of nonempty compact subsets of $Y$, satisfy $\{x: F(x) \cap C$ $\neq \varnothing\} \in \mathbf{H}$ for any closed subset $C$ of $Y$. Then $F$ admits a $\left(\mathbf{H} \cap \mathbf{H}^{c}\right)_{\lambda^{-}}$ measurable selector.
\end{abstract}

1. Introduction. Let $X$ be any set, $\mathbf{H}$ a family of subsets of $X$ and $\tau$ any cardinal. We say that $\mathbf{H}$ is $\tau$-addiiive ( $\tau$-multiplicative) if whenever $\left\{A_{\alpha}\right.$ : $\alpha<\beta\} \subseteq \mathbf{H}$, where $\beta<\tau, \cup_{\alpha<\beta} A_{\alpha}\left(\cap_{\alpha<\beta} A_{\alpha}\right) \in \mathbf{H}$. $\mathbf{H}^{c}$ is the family of subsets of $X$ whose complements belong to $\mathbf{H}$ and $\mathbf{H}_{\tau}$ is the smallest $\tau$-additive family containing $\mathbf{H}$. $\mathbf{H}$ is said to satisfy the $\tau$-weak reduction principle $\left(\tau\right.$-WRP) if given $\left\{A_{\alpha}: \alpha<\beta\right\} \subseteq \mathbf{H}$, such that $\cup_{\alpha<\beta} A_{\alpha}=X$, where $\beta<\tau$, there exists a pairwise disjoint family of sets $\left\{B_{\alpha}: \alpha<\beta\right\} \subseteq \mathbf{H}$ satisfying $B_{\alpha} \subseteq A_{\alpha}$ for all $\alpha$ and $\cup_{\alpha<\beta} B_{\alpha}=X . \aleph_{0}$ and $\aleph_{1}$ are used to denote the first infinite ordinal and the first uncountable ordinal respectively. (Note that cardinals are considered as initial ordinals.)

If $X$ is any set, $\mathbf{H}$ a family of subsets of $X$ and $Y$ a topological space, then a function $f$ on $X$ into $Y$ is called $\mathbf{H}$-measurable if $f^{-1}(U) \in \mathbf{H}$ for every open subset $U$ of $Y . f$ is called a selector for a multifunction $F$ on $X$ into the family of nonempty subsets of $Y$ if $f(x) \in F(x)$ for all $x \in X$. If $A \subseteq X \times Y$ for any sets $X, Y$, then $A^{x}$ denotes the subset of $Y$ given by $\{y:(x, y) \in A\} . \Pi_{1}$ denotes the projection to the first coordinate on $X \times Y$.

In this paper we prove the following:

THEOREM. Let $X$ be any set, $\mathbf{H}$ a family of subsets of $X$ which is $\lambda$-additive, $\lambda$-multiplicative and satisfies the $\lambda$-WRP for some cardinal $\lambda>\boldsymbol{\aleph}_{0}$. Suppose $Y$ is a regular Hausdorff space of topological weight $\leqslant \lambda$ such that given any family of open sets in $Y$, there is a subfamily of cardinality $<\lambda$ with the same union. Let $F: X \rightarrow \mathbf{C}(Y)$, where $\mathbf{C}(Y)$ is the family of nonempty compact subsets of $Y$, satisfy $\{x: F(x) \cap C \neq \varnothing\} \in \mathbf{H}$ for any closed subset $C$ of $Y$. Then $F$ admits a $\left(\mathbf{H} \cap \mathbf{H}^{c}\right)_{\lambda}$-measurable selector.

Received by the editors October 12, 1977.

AMS (MOS) subject classifications (1970). Primary 04A05; Secondary 54C60, 54C65.

Key words and phrases. $\tau$-additive family of sets, $\tau$-multiplicative family of sets, $\tau$-weak reduction principle, $\mathbf{H}$-measurable function, selector. 
By putting $\lambda=\aleph_{1}$ we can deduce the following theorem of Sion: Let $\mathbf{H}$ be a family of subsets of a set $X$ and $Y$ a regular $T_{1}$ space of topological weight $\leqslant \aleph_{1}$ such that each family of open subsets of $Y$ admits a countable subfamily with the same union. Let $F: X \rightarrow \mathbf{C}(Y)$ be such that $\{x: F(x) \cap C$ $\neq \varnothing\} \in \mathbf{H}$ for every closed set $C$ in $Y$. Then $F$ admits a $\sigma(\mathbf{H})$-measurable selector where $\sigma(\mathbf{H})$ denotes the smallest $\sigma$-algebra containing $\mathbf{H}$.

\section{Proof.}

Lemma. Let $X, Y, \mathbf{H}$ and $F$ be as in the theorem. Let $\left\{U_{\alpha}: \alpha\right.$ is a successor ordinal $<\lambda\}$ be an open base for $Y$ such that $U_{\alpha} \neq \varnothing$ for any $\alpha$. Then there exists a family $\left\{A_{\alpha}: \alpha<\lambda\right\}$ of subsets of $X \times Y$ satisfying the following:

(i) For each $\alpha$ and $x, \varnothing \neq A_{\alpha}^{x} \subseteq F(x)$ and $A_{\alpha}^{x}$ is compact.

(ii) For each $\alpha,\left\{x: A_{\alpha}^{x} \cap C \neq \varnothing\right\} \in \mathbf{H}$ if $C \subseteq Y$ is closed.

(iii) If $\alpha<\beta, A_{\beta} \subseteq A_{\alpha}$ for all $\alpha$ and $\beta$.

(iv) If $\alpha$ is a successor ordinal, then there exists $B_{\alpha} \in \mathbf{H} \cap \mathbf{H}^{c}$ such that

$$
\left(X \times \bar{U}_{\alpha}\right) \cap A_{\alpha}=\left(B_{\alpha} \times \bar{U}_{\alpha}\right) \cap A_{\alpha}=\left(B_{\alpha} \times Y\right) \cap A_{\alpha} .
$$

Proof. We define the $A_{\alpha}$ 's by induction as follows: $A_{0}=\cup_{x}(\{x\} \times$ $F(x)$ ). Suppose $A_{\beta}$ is defined for all $\beta<\alpha$.

Case $1 . \alpha=\beta+1$ for some $\beta$.

For any successor ordinal $\gamma$, let $D_{\gamma}^{\beta}=\left\{x: A_{\beta}^{x} \cap \bar{U}_{\gamma} \neq \varnothing\right\}$. By induction hypothesis, $\underline{D}_{\gamma}^{\beta} \in \mathbf{H}$. Now $X-\bar{U}_{\beta+1}=\cup\left\{\bar{U}_{\gamma}: \bar{U}_{\gamma} \subseteq X-\bar{U}_{\beta+1}\right\}=\cup\left\{U_{\gamma}\right.$ : $\left.\bar{U}_{\gamma} \subseteq X-\bar{U}_{\beta+1}\right\}$. Let $\left\{U_{\gamma}: \gamma \in \Gamma_{\beta}\right\}$ be a subfamily of $\left\{U_{\gamma}: \bar{U}_{\gamma} \subseteq X-\right.$ $\left.\vec{U}_{\beta+1}\right\}$ such that cardinality of $\Gamma_{\beta}<\lambda$ and $\bigcup\left\{U_{\gamma}: \gamma \in \Gamma_{\beta}\right\}=\bigcup\left\{U_{\gamma}: \bar{U}_{\gamma} \subseteq\right.$ $\left.X-\bar{U}_{\beta+1}\right\}$. Thus

$$
\bigcup_{\gamma \in \Gamma_{\beta}} U_{\gamma}=\bigcup_{\gamma \in \Gamma_{\beta}} \bar{U}_{\gamma}=X-\bar{U}_{\beta+1} .
$$

If $x \notin D_{\beta+1}^{\beta}, A_{\beta}^{x} \cap \bar{U}_{\beta+1}=\varnothing$ and hence $A_{\beta}^{x} \cap \bar{U}_{\gamma} \neq \varnothing$ for some $\gamma \in \Gamma_{\beta}$. Hence $x \in D_{\gamma}^{\beta}$ for some $\gamma \in \Gamma_{\beta}$. Thus $X=D_{\beta+1}^{\beta} \cup\left\{D_{\gamma}^{\beta}: \gamma \in \Gamma_{\beta}\right\}$. By $\lambda$-WRP of $\mathbf{H}$, find a pairwise disjoint family of sets $B_{\beta+1}^{\beta},\left\{B_{\gamma}^{\beta}: \gamma \in \Gamma_{\beta}\right\}$ in $\mathbf{H}$

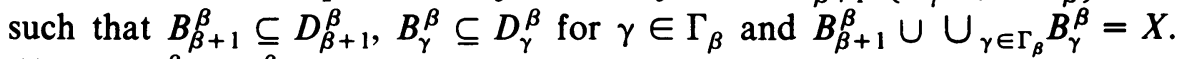
Clearly, $B_{\beta+1}^{\beta}, B_{\gamma}^{\beta} \in \mathbf{H} \cap \mathbf{H}^{c}$.

Define

$$
A_{\beta+1}=\left(\left(B_{\beta+1}^{\beta} \times \bar{U}_{\beta+1}\right) \cup \bigcup_{\gamma \in \Gamma_{\beta}}\left(B_{\gamma}^{\beta} \times \bar{U}_{\gamma}\right)\right) \cap A_{\beta}
$$

and $B_{\beta+1}=B_{\beta+1}^{\beta}$. (i), (iii) and (iv) are clearly satisfied. To check (ii), let $C \subseteq Y$ be closed. Then $\left\{x: A_{\beta+1}^{x} \cap C \neq \varnothing\right\}=\left\{x: x \in B_{\beta+1}^{\beta}\right.$ and $A_{\beta}^{x} \cap$ $\left.\bar{U}_{\beta+1} \cap C \neq \varnothing\right\} \cup \cup_{\gamma \in \Gamma_{\beta}}\left\{x: x \in B_{\gamma}^{\beta}\right.$ and $\left.A_{\beta}^{x} \cap \bar{U}_{\gamma} \cap C \neq \varnothing\right\}$. As $\mathbf{H}$ is $\lambda$-multiplicative (and hence $\kappa_{0}$-multiplicative) and $\lambda$-additive, using the induction hypothesis, we see that $\left\{x: A_{\beta+1}^{x} \cap C \neq \varnothing\right\} \in \mathbf{H}$.

Case 2. $\alpha$ is a limit ordinal.

Let $A_{\alpha}=\cap_{\beta<\alpha} A_{\beta}$. As $\varnothing \neq A_{\beta}^{x} \subseteq F(x)$ for $\beta<\alpha$, each $A_{\beta}^{x}$ is compact 
and $\left\{A_{\beta}^{x}: \beta<\alpha\right\}$ has the finite intersection property by (iii), it follows that $\varnothing \neq A_{\alpha}^{x} \subseteq F(x)$ and $A_{\alpha}^{x}$ is compact. Clearly, (iii) is satisfied and (iv) does not need any verification as $\alpha$ is not a successor ordinal.

To check (ii), let $C \subseteq Y$ be closed.

$$
\begin{aligned}
\left\{x: A_{\alpha}^{x} \cap C \neq \varnothing\right\} & =\left\{x: \bigcap_{\beta<\alpha} A_{\beta}^{x} \cap C \neq \varnothing\right\} \\
= & \left\{x: \bigcap_{\beta<\alpha}\left(A_{\beta}^{x} \cap C\right) \neq \varnothing\right\}=\bigcap_{\beta<\alpha}\left\{x: A_{\beta}^{x} \cap C \neq \varnothing\right\} .
\end{aligned}
$$

The last equality is obtained by using the compactness of $A_{\beta}^{x} \cap C, \beta<\alpha$. As $\alpha<\lambda$ and $\left\{x: A_{\beta}^{x} \cap C \neq \varnothing\right\} \in \mathbf{H}$ for $\beta<\alpha$ by induction hypothesis, $\{x$ : $\left.A_{\alpha}^{x} \cap C \neq \varnothing\right\} \in \mathbf{H}$ by $\lambda$-multiplicativity of $\mathbf{H}$.

This completes the proof of the lemma.

Proof of THE THeOREM. Let $U_{\alpha}, B_{\alpha}, \alpha$ is a successor ordinal $<\lambda$ and $A_{\alpha}$, $\alpha<\lambda$, be as in the lemma. Put $G=\bigcap_{\alpha<A} A_{\alpha}$.

Step 1. $G$ is the graph of a function $f$ and $f$ is a selector for $F$.

By (i) and (iii), $\varnothing \neq G^{x} \subseteq F(x)$ for all $x$. We show that for all $x, G^{x}$ is a singleton. If not, let there exist points $(x, y),(x, z)$ in $G$ where $y \neq z$. Find a basic open set $U_{\alpha} \subseteq Y$ such that $y \in U_{\alpha} \subseteq \bar{U}_{\alpha} \subseteq X-\{z\}$. As $(x, y) \in G \subseteq$ $A_{\alpha}$, it follows that $(x, y) \in\left(X \times \bar{U}_{\alpha}\right) \cap A_{\alpha}=\left(B_{\alpha} \times \bar{U}_{\alpha}\right) \cap A_{\alpha}$, $\alpha$ being a successor ordinal. Thus $x \in B_{\alpha}$ and hence $(x, z) \in\left(B_{\alpha} \times Y\right) \cap A_{\alpha}=\left(B_{\alpha} \times\right.$ $\left.\bar{U}_{\alpha}\right) \cap A_{\alpha}$. Therefore $z \in \bar{U}_{\alpha}$ which is a contradiction. Define $f(x)=y$ if $\{y\}=G^{x}$.

Step 2. We now have to show that the function $f: X \rightarrow Y$ is $\left(\mathbf{H} \cap \mathbf{H}^{c}\right)_{\lambda^{-}}$ measurable.

Let $V \subseteq Y$ be open. $V=\bigcup\left\{\bar{U}_{\alpha}: \bar{U}_{\alpha} \subseteq V\right\}=\bigcup\left\{U_{\alpha}: \bar{U}_{\alpha} \subseteq V\right\}$. There exists a subfamily $\left\{U_{\alpha}: \alpha \in \Gamma\right\}$ of $\left\{U_{\alpha}: \bar{U}_{\alpha} \subseteq V\right\}$ such that cardinality of $\Gamma<\lambda$ and $\cup_{\alpha \in \Gamma} U_{\alpha}=V$. Thus $\cup_{\alpha \in \Gamma} \bar{U}_{\alpha}=V$. Hence

$$
f^{-1}(V)=\bigcup_{\alpha \in \Gamma} f^{-1}\left(\bar{U}_{\alpha}\right)=\bigcup_{\alpha \in \Gamma}\left(\Pi_{1}\left(\left(X \times \bar{U}_{\alpha}\right) \cap G\right)\right) \text {. }
$$

It is enough to show that $\Pi_{1}\left(\left(X \times \bar{U}_{\alpha}\right) \cap G\right) \in \mathbf{H} \cap \mathbf{H}^{c}$ for any successor ordinal $\alpha$.

Fix $\alpha$.

$$
\Pi_{1}\left(\left(X \times \bar{U}_{\alpha}\right) \cap G\right)=\Pi_{1}\left(\left(X \times \bar{U}_{\alpha}\right) \cap \bigcap_{\gamma<\lambda} A_{\gamma}\right) .
$$

We first note the $\Pi_{1}\left(\left(X \times \bar{U}_{\alpha}\right) \cap \cap_{\gamma<\lambda} A_{\gamma}\right)=\bigcap_{\gamma<\lambda} \Pi_{1}\left(\left(X \times \bar{U}_{\alpha}\right) \cap A_{\gamma}\right)$. Clearly,

$$
\Pi_{1}\left(\left(X \times \bar{U}_{\alpha}\right) \cap \bigcap_{\gamma<\lambda} A_{\gamma}\right) \subseteq \bigcap_{\gamma<\lambda} \Pi_{1}\left(\left(X \times \bar{U}_{\alpha}\right) \cap A_{\gamma}\right) .
$$

Let $x \in \cap_{\gamma<\lambda} \Pi_{1}\left(\left(X \times \bar{U}_{\alpha}\right) \cap A_{\gamma}\right)$. Then for all $\gamma<\lambda, A_{\gamma}^{x} \cap \bar{U}_{\alpha} \neq \varnothing$. As 
$A_{\gamma}^{x} \cap \bar{U}_{\alpha}$ is compact for each $\gamma<\lambda$, using (iii), we see that $\cap_{\gamma<\lambda}\left(A_{\gamma}^{x} \cap \bar{U}_{\alpha}\right)$ $\neq \varnothing$ so that $x \in \Pi_{1}\left(\left(X \times \bar{U}_{\alpha}\right) \cap \cap_{\gamma<\lambda} A_{\gamma}\right)$.

Again, using (iii), we obtain

$$
\bigcap_{\gamma<\lambda} \Pi_{1}\left(\left(X \times \bar{U}_{\alpha}\right) \cap A_{\gamma}\right)=\bigcap_{\alpha<\gamma<\lambda} \Pi_{1}\left(\left(X \times \bar{U}_{\alpha}\right) \cap A_{\gamma}\right) .
$$

Hence $\Pi_{1}\left(\left(X \times \bar{U}_{\alpha}\right) \cap G\right)=\bigcap_{\alpha \leqslant \gamma<\lambda} \Pi_{1}\left(\left(X \times \bar{U}_{\alpha}\right) \cap A_{\gamma}\right)$. We next prove that

$$
\bigcap_{\alpha<\gamma<\lambda} \Pi_{1}\left(\left(X \times \bar{U}_{\alpha}\right) \cap A_{\gamma}\right)=\Pi_{1}\left(\left(X \times \bar{U}_{\alpha}\right) \cap A_{\alpha}\right)
$$

Clearly, $\cap_{\alpha \leqslant \gamma<\lambda} \Pi_{1}\left(\left(X \times \bar{U}_{\alpha}\right) \cap A_{\gamma}\right) \subseteq \Pi_{1}\left(\left(X \times \bar{U}_{\alpha}\right) \cap A_{\alpha}\right)$. Let

$$
x \in \Pi_{1}\left(\left(X \times \bar{U}_{\alpha}\right) \cap A_{\alpha}\right)=\Pi_{1}\left(\left(B_{\alpha} \times Y\right) \cap A_{\alpha}\right)
$$

(by (iv)). Then $x \in B_{\alpha}$ and hence $A_{\alpha}^{x} \subseteq \bar{U}_{\alpha}$. If $\alpha \leqslant \gamma<\lambda, \varnothing \neq A_{\gamma}^{x} \subseteq A_{\alpha}^{x} \subseteq$ $\bar{U}_{\alpha}$ so that $A_{\gamma}^{x} \cap \bar{U}_{\alpha} \neq \varnothing$. Hence $x \in \Pi_{1}\left(\left(X \times \bar{U}_{\alpha}\right) \cap A_{\gamma}\right)$ for $\alpha \leqslant \gamma<\lambda$.

Thus

$$
\begin{aligned}
\Pi_{1}\left(\left(X \times \bar{U}_{\alpha}\right) \cap G\right) & =\Pi_{1}\left(\left(X \times \bar{U}_{\alpha}\right) \cap A_{\alpha}\right) \\
& =\Pi_{1}\left(\left(B_{\alpha} \times Y\right) \cap A_{\alpha}\right)=B_{\alpha} \in \mathbf{H} \cap \mathbf{H}^{c}
\end{aligned}
$$

Corollary. If $X, Y, \mathbf{H}$ are as in the theorem and if $F: X \rightarrow \mathbf{C}(Y)$ is such that $\{x: F(x) \cap U \neq \varnothing\} \in \mathbf{H}$ for any open $U \subseteq Y$, then $F$ admits $a(\mathbf{H} \cap$ $\left.\mathbf{H}^{c}\right)_{\lambda}$-measurable selector.

Proof. Let $\left\{U_{\alpha}: \alpha<\lambda\right\}$ be a base for $Y$ consisting of nonempty open sets and let $C \subseteq Y$ be closed. Then $Y-C=\cup\left\{\bar{U}_{\alpha}: \bar{U}_{\alpha} \subseteq Y-C\right\}=\cup\left\{U_{\alpha}\right.$ : $\left.\bar{U}_{\alpha} \subseteq Y-C\right\}$. Let $\left\{U_{\alpha}: \alpha \in \Gamma\right\}$ be a subfamily of $\left\{U_{\alpha}: \bar{U}_{\alpha} \subseteq Y-C\right\}$ which has cardinality $<\lambda$ and satisfying $\cup_{\alpha \in \Gamma} U_{\alpha}=Y-C$. Clearly, $\cup_{\alpha \in \Gamma} \bar{U}_{\alpha}=$ $Y-C$.

Now as $F(x)$ is compact, $F(x) \subseteq Y-C$ if and only if there exist $\alpha_{1}, \ldots, \alpha_{n} \in \Gamma$ such that $F(x) \subseteq \bigcup_{i=1}^{n} U_{\alpha_{i}} \subseteq \cup_{i=1}^{n} \bar{U}_{\alpha_{i}} \subseteq Y-C$. Thus

$$
\begin{aligned}
\{x: F(x) \subseteq Y-C\} & =\bigcup_{n} \bigcup\left\{x: F(x) \cap \bigcap_{i=1}^{n} \bar{U}_{\alpha_{i}}^{c}=\varnothing\right\} \\
& \in\left(\left(\mathbf{H}^{c}\right)_{\lambda}\right)_{\alpha_{1}}=\left(\mathbf{H}^{c}\right)_{\lambda}=\mathbf{H}^{c}
\end{aligned}
$$

as $\mathbf{H}$ is $\lambda$-multiplicative. Hence $\{x: F(x) \cap C \neq \varnothing\} \in \mathbf{H}$. Now we can invoke the theorem.

3. Acknowledgement. I am grateful to Dr. Ashok Maitra for his suggestions.

\section{REFERENCES}

1. A. Maitra and B. V. Rao, Selection theorems for partitions of Polish spaces, Fund. Math. 93 (1976), 47-56.

2. M. Sion, On uniformization of sets in topological spaces, Trans. Amer. Math. Soc. 96 (1960), 237-245.

Statistics and Mathematics Division, Indian Statistical Institute, Calcutta 700035 , INDIA 